\title{
Health Care and Pharmaceuticals 2020: Key factors, expected trends and way ahead
}

\section{Dr Varsha Narayanan}

Health and Pharmaceutical Consultant, Dr Varsha's Health Solutions, Andheri west, Mumbai.

Email: info@drvarsha.com web: www.drvarsha.com

\begin{abstract}
Health Care and Pharmaceuticals in India are two big segments which are fast growing and influenced by population, environmental, economic and technological factors. 2020 and the following decade will see innovation, expansion and increased effectiveness of these sectors. For Health Care: patient empowerment, strengthening peripheral health services and quality of care, an integrated therapeutic approach, along with leveraging big data analytics and technological advancements, will play vital roles in significantly enhancing and expanding health care in India. The collaboration of Pharmaceutical Industry with Health care and its practitioners will assume significant importance. Key factors like real world evidence and data, evidence based communication and rational promotion, Focus on drug safety and pharmacovigilance, scientific collaborations with key opinion leaders for updating and disseminating scientific knowledge to improve clinical decision making and patient outcomes, and all this coupled with an innovative pipeline will define the credible, long-term and prominent pharmaceutical players.
\end{abstract}

KEY WORDS: Health Care, Pharmaceuticals, Real world evidence, Big data, AI, Technology, innovation

\section{INTRODUCTION}

As we have entered the year 2020, it is apt to review the current size, and take a look at the possible factors and trends which would influence and shape the healthcare and pharmaceutical industry in the decade to come.

The global healthcare market which includes healthcare services and pharmaceutical drugs (the former contributing 80\%) reached a value of nearly $\$ 8.5$ trillion in 2018, having grown at a compound annual growth rate (CAGR) of $7.3 \%$ since 2014, and is expected to grow at a CAGR of $8.9 \%$ to nearly $\$ 11.9$ trillion by $2022 .{ }^{1}$ The healthcare services and pharmaceutical drug markets are expected to grow at CAGRs of $9.3 \%$ and $6 \%$ respectively.

The fastest growing regions in the healthcare market will be the Asia Pacific and Africa, where CAGRs of $13.4 \%$ and $13.1 \%$ respectively are expected to be seen.Healthcare in India was a US\$ 61.79 billion in 2017 and is expected to reach US\$ 132.84 billion by $2023 .{ }^{2}$ In 2020 , the value of pharmaceutical products sold in the India is expected to reach $\$ 55$ billion, with double-digit growth in the coming decade. ${ }^{3}$

\section{HEALTH CARE}

Health care in India represents an eclectic range from the need for access to basic health care and more peripheral health services, to having the most advanced facilities and technologies 
at apex and tertiary care institutes. The 5 factors envisioned to play vital roles in shaping health care in India, as well as in most developing economies worldwide would be: Patient empowerment, Strengthening Peripheral Health Services and Quality of care, Integrated therapeutic approach, using Big Data Analytics to improve Health care, and leveraging the Technological boom.

\section{Patient Empowerment}

Patients today have much more access to health related information due to penetration of internet.Asia has an internet penetration of $54.2 \%$ which represents $50 \%$ of the world's population. ${ }^{4}$ India's internet users have registered double digit $18 \%$ growth to reach 627 million in 2019representing a $40 \%$ overall internet penetration. ${ }^{5} 87 \%$ of these are defined as regular users, having accessed internet in last 30 days. Nearly 200 million active internet users reside in rural India. ${ }^{5}$

The patient or consumer today is thus far more aware,due to all kinds of health information available at the fingertip. However, 'empowerment' comes from convenient access to credible, and relevant health information. Therefore, sources of information would have to come from credible sources like doctors with documented medical background, experience and scientific acclaimed work, or from recognized medical associations, clinics or institutes. These sources should also carry with them inbuilt online facility or remote-access for queryinteraction. The active role played by Health Care Providers (HCPs) in empowering patients with right health information and guidance can be one of the keys to successful patient outcomes and satisfaction.

\section{Strengthening Peripheral Health Services and Quality of care}

As well known the top of the pyramid cannot stand without a strong base. Peripheral health care services and centers, which cater to the major vast population in developing countries form the health care back bone which needs continuous strengthening. Presently there are around 24,000 PHCs (Primary Health Centers) in India, each having one doctor (General physician - health officer) along with nurse and health workers, catering to a population of 20,000 to $30,000^{6}$ Around 4 PHCs are mapped to the secondary level of care (First referral units) called CHCs (Community Health Centers) which have pediatric and gynecological specialists (and sometimes dentists) in addition to general physicians. The 5000 odd CHCs in India are mapped to only around 600 district hospitals and further to 200 odd tertiary care government hospitals. ${ }^{7}$

The health-care focus for the 2020 onward decade has to be on Family and Community Medicine, whereinthe development and strengthening of the PHCs and CHCs could be enhanced by participation from private specialty hospitals in towns and cities. There are currently around 2500 such big advanced specialty/super-specialty hospitals in the country, which can pioneer a wave of peripheral health care enhancement by taking up a CHC and its mapped PHCs under their wing. This will not only improve facilities but also increase cascading of knowledge, updating, training and educatingperipheral doctors, nurses and health workers on basic patient care in various therapeutic and disease segments. This will give a 3-fold advantage of improving quality of careand effectiveness, facilitate accountability and monitoring, and giveperipheral doctors exposure to changing global concepts in disease management and new drugs which will also encourage more medical graduates to take up such peripheral positions. ${ }^{8}$ 


\section{Integrated therapeutic approach}

Health care has majorly focused on prescription and interventional medicine, with streamlining and segregation of allopathic, and herbal/traditional medical practice. Today there is great awareness and recognition of Dietary and Lifestyle factors in diseases. Several herbs have now through clinical trials been approved for use in allopathic medicine as supplements and drugs. Therefore, most diseases today require a holistic and integrated therapeutic approach, which should complement drug therapy with lifestyle, nutritional/dietary, psychosocial and scientific indigenous/alternative therapies, the last having an important place in rural health care in developing nations like India.

The Indian healthcare system at the peripheral primary, community and district level is soon going to see integrated allopathic and alternate medicine services in 50-100\% centers by 2023. ${ }^{9}$ Centers of Integrated medicine will also be set up in tertiary care teaching hospitals, a process already initiated. For management of most chronic diseases, the trends in superspecialty hospitals will also shift increasingly to team based integrated and holistic therapy with lifestyle modification, diet structuring, natural supplements and psychological consideration being important aspects of disease management for effective symptomatic relief as well as long term benefit. ${ }^{10}$ Aims of therapy will trend towards Holistic Health and patient Wellness, and this multidimensional approach will define game changers in managing patients with Chronic diseases.

\section{Big Data Analytics}

The health care industry is the source of huge quantities of information that are now consolidated and analyzed through the digitization of records. Stratification and consolidation of this data and its analysis can help in understanding disease patterns, demographic and epidemiological trends, patient risk factors and predispositions, seasonal and regional variations, symptom patterns, comorbidities and associations, treatment trends and response, disease prognosis, patient compliance and drug safety/adverse events. ${ }^{11}$

Big data analysis can thus improve disease prediction and prevention, timely symptom recognition and diagnosis, as well as aid in predicting disease prognosis, and reducing complications. In addition to its obvious benefit in patient disease management, big data analysis can also help in better organization, improved efficiency and cost reduction for hospitals and clinics of almost $25 \%$ reduction over a 5 -year period. It also improves patient engagement, convenience and monitoring, More and more health care providing centers are moving to EHR (Electronic Health records) which are used to study the patient, disease and response trends of the individual center as well as is pooled into the data lakes for larger regional and national predictive analysis. Efficient barriers of patient anonymity and confidentiality are vitally integrated, making big data analytics an ethical and pertinent research tool for the advancement and effectiveness of health care in any country. ${ }^{12}$

\section{Leveraging the Technological boom}

Advancements and Innovations in Technology for Diagnosis and Treatment have always been quickly embraced and adapted to by the health care industry. In diagnosis the forthcoming decade will continue tofurther move towards non-invasive/minimally invasive diagnostic techniques, decreasing duration of tests and its results (rapid/spot diagnostic tests), increasing diagnostic precision, sensitivity and specificity through new technology or upgraded versions, new tests for early diagnosis and screening, home/remote/in-clinic 
diagnostic and screening modalities, and more diagnostic-interventional approaches in same sitting to save patient time, and cost. Surgical advancements with improved tools, lasers, bioimplants, and techniques will greatly improve patient outcomes and recovery time.

\section{AI in health care}

The most significant technological revamping of the health care system is predicted to be through Artificial Intelligence (AI) and Remote/Telemedicine. The application of AI will be seen in improving and revolutionizing mainly 3 areas of Health care: Diagnosis and Treatment, Patient engagement and adherence, and administrative efficiency and applications. ${ }^{13,14}$

AI mainly functions through machine learning, neural network and deep learningwhich can help in designing treatment strategies and predicting outcomes based on various patient factors, evaluate patient risk for any disease and increase diagnostic precision like recognition of potentially cancerous lesions in radiology images, or the detection of clinically relevant features in imaging data beyond human eye perception.

NLP (Natural Language Processing) includes applications such as speech recognition, text analysis, translation and other language skills for the creation, understanding and classification of clinical documentation, analyzing unstructured clinical notes on patients, preparing reports, transcribing patient interactions and conducting conversations in the form of patient support or assistant chat bots.Messaging alerts, relevant-targeted content and aiding patient self-monitoring can initiate and modify human behavior and actions at important junctures, positively affecting the overall treatment response. Patient data collected, comparison and analysis from EHR systems, biosensors, watches, smartphones, conversational interfaces and other instrumentation software can help customize recommendations and follow-ups.

Robotics, which increases surgical precision, rises above the human limits of vision, angulation and finesse, and reduces surgical, hospitalization and healing time, is poised to take over the surgical space in a big way. Soon Robots with AI-based 'brains' will lead to image recognition is being integrated with Robotic process automation (RPA).

It is important to note here that AI systems will not replace human clinicians but enhance the patient care they can impart which can also help clinicians and HCPs to focus on and increase time spent on aspects requiring uniquely human skills like empathy, persuasion, integrating the big-picture and effective clinical decision making.

\section{PHARMACEUTICAL ROLE IN HEALTH CARE}

The role of the pharmaceutical industry is fast changing from being a producer and supplier of medicines to being an active part of medical research, medical knowledge updating, therapy shaping and enhancing patient care. Going ahead, the following will play critical roles in enabling the pharmaceutical industry towork hand in hand with the health care providers and medical practitioner in continuously improving patient treatment outcomes: Real world evidence and data generation, Rational promotion and evidence based communication, Drug safety monitoring and updating, Scientific collaborations with HCPs and Innovative pipeline.

\section{Real World Evidence and Data}

All drug launches happen after randomized controlled trials (RCTs) have establish efficacy and safety in an appropriately sized population. Real World Evidence (RWE) is derived from the analysis of data collected from healthcare settings, outside the ambit of the strict patient selection criteria of RCTs, and helps to understand observations and events in different kinds 
of patients in routine clinical practice. RWE complements RCTs, which are carefully controlled studies testing specific hypotheses (endpoints) on the efficacy and safety of new drugs, and are not designed to represent responses in day to day or real world clinical practice. $^{15}$

The US FDA aims to publish draft guidance for the use of RWE by October 2021, In Asia, (including India), there is growing awareness among regulatory authorities to structure the inclusion of real world evidence into assessing long term efficacy and safety of approved drugs in different patient groups and its benefit in clinical and economical decision making. Though RWE generation is focused mainly on post launch assessment of drugs, soon earlier stages of the clinical drug development pipeline may also start to use RWE to support critical decisions. Now with companies specializing in generating RWE for the pharmaceutical industry, the trend is increasingly going to shift towards RWE based promotion and communication. ${ }^{16}$ RWE will also be an important factor for several herbal and nutritional supplements which have been launched for allopathic co-prescriptions in many chronic diseases.

The HCPs and the pharmaceutical industry have a symbiotic relationship in RWE generation with the former helping in effective data generation in clinical practice and the latter designing its capture, regular and effective analysis and sharing of inferences through scientific meets, publications and communication with HCPs. The HCPs use the inferences for customizing treatment strategies and optimizing patient outcomes. ${ }^{15}$

\section{Rational promotion and evidence based communication}

This is an important aspect which can favorably impact the long term growth and credibility of both the pharmaceutical industry and the HCPs as well as have bearings on drug safety/adverse events, patient outcomes and pharmacoeconomics. There is a constant interplay between company pressures and short term sales target, and indication, scientific guidelines and clinical evidence based promotion, however regulators worldwide along with World health organization are moving towards suggesting and slowly enforcing the same. ${ }^{17}$

The problem of irrational medicine use is particularly known to be worse in developing countries with weak health systems, where mechanisms for routine monitoring of medicine use are often not well developed or are at times non-existent. ${ }^{18}$ Certain category of medicines like antibiotics, pain medications (NSAIDs, opioids), Drugs for anxiety/depression and sleep, Corticosteroids, immunomodulators and cough/cold medications have been under scrutiny for promotion in inappropriate indications, lack of adequate evidence/studies and increased usage in milder-self resolving conditions or instead of other conservative/non pharmacological remedies. Fixed dose combinations without clinical data of clear efficacy/safety over monotherapy or available RWE of its benefit have also recently been discouraged by regulators.

The future and long term key players in the pharmaceutical drug market will emerge from those with rational promotion based on clinical trial evidence as well as RWE, the latter being the game changer. Case based scenario approach in communication as well as regular drug safety/pharmacovigilance updates will be seen as added values for prescription of the drug and its recommendation in scientific standard clinical consensus and guidelines.Promoting the rational use of medicines requires effective policies as well as efficient collaboration between pharmaceutical companies, health professionals, regulators, patients, and entire communities. ${ }^{18}$

Drug safety monitoring- Pharmacovigilance

To improve the ability of patients to receive high-quality, safe, effective, and timely care, better information via pharmacovigilance will be a priority in the decades to come for the 
regulators, HCPs and patients. Digitization and electronic records and regular generation of RWE as well as post marketing studies can harness drug safety data effectively along with individual reporting by HCPs.

Developing validated tools and techniques for "predictive pharmacovigilance" will help health systems in better understanding of the risks and benefits of drugs/medicines as to the journey of a new medicine from risk-benefit regulatory efficacy to real-world riskeffectiveness and tolerance. ${ }^{19}$ This will aid in comparing preapproval predictive pharmacovigilance data, to better understand the potential gap between what was predicted and what was actually measured in the real world scenario. The focus of pharmacovigilance needs to shift from a regulatory compulsion and exercise to an essential part of scientific communication, and building RWE. ${ }^{20}$

One of the need gaps is the disconnect between the field force and medical representatives driving promotion, communication and prescription with HCPs, and the drug monitoring and PV systems within the company. Integrating the two via Medical affairs and Medicomarketing professionals can help bridge this gap, and change the perception of pharmacovigilance being a hurdle to one of being a value add for scientific brand building.

\section{Scientific Collaboration with Key Opinion Leaders (KOLs) and HCPs}

The Health care world is a dynamic and constantly evolving space with newer and newer data, drugs and treatment modalities coming in frequently. This leads to need for constant knowledge updating; modifying, optimizing and customizing treatment strategies, guidelines and recommendations; and cascading the same effectively down to the periphery. The role of collaborations between Health Care centers, Apex institutes, Key opinion leaders and the HCPs including those at peripheral level, cannot be emphasized enough.

More and more pharmaceutical resource allocation is being seen in conduction of such scientific advisory meetings for discussing and analyzing changing and new therapies and treatment concepts; preparation and cascading of consensus, teaching modules and treatment recommendation/algorithms to HCPs across the country; supporting skill upgradation workshops for both experience and freshly graduated HCPs; Enhancing academic sessions at conferences through data and technological support; and last but not the least participate actively in enhancing patient screening, diagnosis and outcomes. ${ }^{21}$ The last is especially important as pharmaceutical support can be effectively harnessed for monitoring and improving drug compliance and assisting physicians in increasing patient awareness.

\section{Innovative pipeline}

The future is moving from symptomatic to etiological management as well as preventive medicine which has already heralded the era of biopharmaceuticals (Biologicals and Biosimilars)for several tumors, and chronic inflammatory and degenerative diseases. Further, other different types of innovative therapies likeCell and Gene therapy(CGT) products, Immune-modulation agents, CAR-T (Chimeric Antigenic Receptor therapy) for cancers and autoimmune diseases, harnessing therapeutic benefits of natural substances in food, soil, herbs and animal sources, and using phage therapy to combat antibiotic resistance, are some that are likely to see positive study results and therapeutic usage. ${ }^{22}$

Innovation in drug delivery and improving patient compliance is going to be a thrust area with particle size reduction and improved absorptive properties like nanosphere technology, alternate delivery routes for many injectable drugs like nasal/dermal/mouth dissolving/conjunctival and inhalationaldelivery routes for many injectable drugs, targeted drug delivery with sensors, matrix technology to extend duration of drug action, and other NDDS (Novel drug delivery systems). 
There are also several pipeline new drugs in existing drug classes with multi-prong mechanism of action and better pharmacokinetic profile and tolerance for improving patient compliance, and treatment outcomes for many of the acute and chronic diseases. On the other hand, more economic generic versions of innovator drugs will also continue to add to the growth of Indian and Multinational pharmaceutical companies both domestically and through export.

\section{Conclusion}

The focus in Health Care in developing economies is on Family and Community Health which involves enhancement from the base to the apex with strengthening of peripheral health centers. Symptomatic and segregated approach has now given way to holistic and integrated therapeutic approach with emphasis on long term wellness. Patient empowerment, and engagement are now important components of disease management while data analytics and real world evidence will increasingly guide treatment strategy and clinical decisions.

The 2020 decade ushers in a period of growth, expansion and innovation in both the Health Care and Pharmaceutical space, with more scientific collaboration for enhancing clinical decision making, customized and holistic treatment strategies, patient engagement-adherence and improved clinical patient outcomes.

\section{References}

1. The \$11.9 Trillion Global Healthcare Market: Key Opportunities \& Strategies (20142022). Accessed Jan 2020 on https://www.businesswire.com/news/home/ 20190625005862/en/11.9-Trillion-Global-Healthcare-Market-Key-Opportunities

2. Challenges in Indian Health Care Industry. Accessed Jan 2020 on https://www.civilserviceindia.com/current-affairs/articles/challenges-in-indianhealthcare-industry.html

3. Indian Pharmaceutical Market Update. Accessed Jan 2020 on https://www.thepharmaletter.com/article/indian-pharma-market-update

4. Internet World Statistics. Accessed Dec 2019 on https://www.internetworldstats.com/stats.htm

5. Kantar IMRB, 21st edition ICUBE Digital adoption \& usage trends:2019. Accessed Dec 2019 on https://imrbint.com/images/common/ ICUBE\%E2\%84\%A2_2019_Highlights.pdf

6. Indian Health Care System. Accessed Jan 2020 on https://mohfw.nic.in/NRHM/Documents/IPHS_for_PHC.pdf

7. Kumar P. How to strengthen Primary Health Care. J Family Med Prim Care. 2016 Jul-Sep; 5(3): 543-546. https://www.ncbi.nlm.nih.gov/pmc/articles/PMC5290757/

8. Ghosh K. Why we don't get doctors for rural medical service in India? The National Medical Journal of India. 2018; $31(1) ; 44-46$ http://www.nmji.in/article.asp?issn=0970258X; year $=2018 ;$ volume $=31 ;$ issue $=1 ;$ spage $=44 ;$ epage $=46 ;$ aulast $=$ Ghosh

9. Integrate AYUSH with Modern Medicine for Holistic Health. Accessed 2020 on https://www.iasparliament.com/current-affairs/integrate-ayush-with-modernmedicine-for-holistic-health

10. Shenwai RS, Tare KN. Integrated Approach Towards Holistic Health: Current Trends and Future Scope. International Journal of Current Research and Review. Apr 2017; 9(7), 11-14 http://ijcrr.com/uploads/280_pdf.pdf 
11. Indian Health Care Analysis using Big Data Programming Tool. Procedia Computer Science. 2016; 89:521-527. https://www.sciencedirect.com/science/article/pii/S1877050916311668

12. Mobar S, Gupta A. Scenario of Analytics in Indian Healthcare.International Journal of Engineering, Business and Enterprise Applications. March-May 2017; 20(1):13-19. https://www.researchgate.net/publication/322713448 Scenario of Analytics in Indi an_Healthcare

13. Jiang F, Jiang Y, Zhi H, et al. Artificial intelligence in healthcare: past, present and future. Stroke and Vascular Neurology 2017;2: e000101. doi:10.1136/svn-2017000101. https://svn.bmj.com/content/2/4/230

14. Davenport T, Kalakota R. The potential for artificial intelligence in healthcare. Future $\begin{array}{lllll}\text { Healthc } & \text { J. } & 2019 & \text { Jun; }\end{array}$ https://www.ncbi.nlm.nih.gov/pmc/articles/PMC6616181/

15. Katkade VB et al. Real world data: an opportunity to supplement existing evidence for the use of long-established medicines in health care decision making. J MultidiscipHealthc. $\quad 2018$; $295-$ 304.https://www.ncbi.nlm.nih.gov/pmc/articles/PMC6033114/

16. Khosla S, White R, Medina J, et al. Real world evidence (RWE) - a disruptive innovation or the quiet evolution of medical evidence generation?. F1000Res. 2018;7:111. https://www.ncbi.nlm.nih.gov/pmc/articles/PMC6039945/

17. Alves TS et al. Medicines Information and the Regulation of the Promotion of Pharmaceuticals. Science and Engineering Ethics. August 2019; 25 (4): 11671192. https://link.springer.com/article/10.1007/s11948-018-0041-5

18. Ofori-Asenso R, Agyeman AA. Irrational Use of Medicines-A Summary of Key Concepts. Pharmacy

(Basel). 2016;4(4):35.https://www.ncbi.nlm.nih.gov/pmc/articles/PMC5419375/

19. Pitts PJ, Le Louet $\mathrm{H}^{-}$Advancing Drug Safety Through Prospective Pharmacovigilance.Ther Innov Regul Sci. 2018 Jul;52(4):400402.https://www.ncbi.nlm.nih.gov/pubmed/29714580

20. Furlan, G., Edwards, B., Mastroianni, A. et al. The Future of Pharmacovigilance: Proposals for More Efficient and Effective Systems-Based Approaches. Pharm Med 2016; 30, 137-142. https://link.springer.com/article/10.1007/s40290-016-0145-7

21. Latten T. Pharmaceutical companies and healthcare providers: Going beyond the gift - An explorative review. PLoS One. 2018; 13(2): e0191856. https://www.ncbi.nlm.nih.gov/pmc/articles/PMC5802853/

22. Pipeline Insights 2020: A Special Report from Pharmaceutical Executive. Accessed 2020

http://files.alfresco.mjh.group/alfresco_images/pharma/2019/11/11/ca513c31-f27a475e-8799-0fe89d5c1ef2/Pharm\%20Exec_2020\%20Pipeline\%20Report.pdf 